\title{
Role of laparoscopy in patients with peritoneal metastases considered for cytoreductive surgery and hyperthermic intraperitoneal chemotherapy (HIPEC)
}

\author{
Thejus T Jayakrishnan', Anthony J Zacharias', Avishkar Sharma', Sam G Pappas², T Clark Gamblin \\ and Kiran K Turaga ${ }^{*}$
}

\begin{abstract}
Background: We hypothesized that diagnostic laparoscopy $(\mathrm{DL})$ was feasible for the evaluation of patients with peritoneal carcinomatosis $(\mathrm{PC})$ undergoing cytoreductive surgery and hyperthermic intraperitoneal chemotherapy (CRS + HIPEC).

Methods: A retrospective review of PC patients treated from January 2010 to April 2013 was conducted. Data on tumor characteristics, treatment details and survival outcomes were extracted and analyzed.

Results: Of the 101 PC patients (mean age $52.9 \pm 14.1$ years), 73 diagnostic laparoscopies DL (61 concurrent with CRS + HIPEC) were performed in 70 patients whereas 31 patients underwent direct exploratory laparotomy (EL). Complete laparoscopic assessment was possible in 63 cases (86.3\%), resulting in 18 exclusions (27.7\%) while 10 cases were converted to open due to inadequate laparoscopic visualization. Subsequently, CRS + HIPEC was performed in $85.4 \%$ (of 55 selected for HIPEC, DL) versus 74.2\% (EL, $P$ value $=0.20$ ). Among those excluded from HIPEC at the initial operation, delayed HIPEC after conversion chemotherapy was achieved in 6 (of 11 with extensive disease, $\mathrm{DL}$ ). The incidence of grade 3 to 5 complications was $0 \% \mathrm{DL}$ versus $10 \% \mathrm{EL}(P$ value $=0.2)$. There were no port site recurrences at mean follow up of $9.1 \pm 8$ months.
\end{abstract}

Conclusions: Laparoscopy is a feasible technique for selecting patients with PC for CRS + HIPEC, and can help select patients for conversion chemotherapy in the setting of high peritoneal carcinomatosis index (PCI) score.

Keywords: Peritoneal neoplasms, regional perfusion, chemotherapy, postoperative complications, surgical procedures, HIPEC, staging, laparoscopy

\section{Background}

Peritoneal carcinomatosis (PC) represents an advanced stage of many cancers and is associated with poor survival rates [1-5]. Cytoreductive surgery (CRS) with hyperthermic intraperitoneal chemotherapy (HIPEC) was introduced in an attempt to improve the prognosis of these patients and incorporates surgical removal of all visible disease followed by chemical destruction of microscopic disease through chemoperfusion [6].

\footnotetext{
*Correspondence: kturaga@mcw.edu

'Division of Surgical Oncology, Department of Surgery, Medical College of Wisconsin, 9200 W Wisconsin Ave, Milwaukee, WI 53226, USA

Full list of author information is available at the end of the article
}

The most validated predictors of outcome in patients undergoing CRS + HIPEC are preoperative tumor burden measured in terms of the peritoneal carcinomatosis index (PCI) and completeness of cytoreduction (CC) [7-10]. Patients undergoing CRS + HIPEC in which surgery achieved complete or near complete cytoreduction (CC-0 or CCR-1) were shown to have significantly greater survival benefit over those who did not $[7,9,11]$. Survival analyses studies have shown that patients with PCI scores less than 19 (colorectal) and 10 (gastric) benefit most from CRS + HIPEC, facilitating the use of these scores as general cut-offs for excluding patients from this morbid procedure [12-15]. Exclusion of 
patients from definitive resection at laparotomy (a nontherapeutic laparotomy) is undesirable and may impede the timely enrollment of patients into alternate therapies [16]. Therefore, the role of preoperative imaging in CRS + HIPEC is to filter out patients with very high volume disease or no carcinomatosis. Despite advancements in cross-sectional imaging, the proportion of patients excluded at direct exploratory laparotomy for CRS + HIPEC is high (20 to 40\%) [17,18].

Diagnostic laparoscopy prior to resection is widely used in hepatopancreaticobiliary and colorectal cancer and has been shown to be effective in excluding unnecessary laparotomy associated with higher morbidity $[19,20]$. The laparoscopy could be done concomitantly at the time of planned resection or as a separate staging procedure [19]. In addition, this may permit earlier enrollment of these patients into palliative or neoadjuvant therapy with potential impact on survival [16]. However, there is a paucity of data on the use of laparoscopy in the evolving field of cytoreductive surgery [21]. Since PC represents an advanced stage of cancer, visualization may be difficult due to adhesions from the cancer or prior surgical procedures. Additionally, there is potentially increased risk of complications such as visceral perforation in the setting of PC. The hypothesis for the present study was that laparoscopic evaluation is feasible for the evaluation of patients with peritoneal carcinomatosis selected for CRS + HIPEC.

\section{Methods}

A retrospective review of patients treated for peritoneal carcinomatosis at the The Froedtert \& the Medical College of Wisconsin's Regional Cancer Therapy Program between January 2010 and April 2013 was performed after approval by the Institutional Review Board (IRB). Demographic information of patients who underwent laparoscopic/laparotomy evaluation for CRS + HIPEC was extracted. Patients who had multiple HIPECS as well as those who underwent CRS outside and were referred to our institute for further management were excluded from the study. Data on tumor characteristics, operative details, hospital course, and morbidity (Clavien-Dindo classification) and survival outcomes were extracted and entered into a pre-specified data extraction sheet. The length of the hospital stay was calculated from the date of the surgical procedure to the date of discharge. The follow-up information current with 4 April 2013 (date of IRB approval) was captured.

All patients referred for treatment under the program underwent routine preoperative computerized tomography $(\mathrm{CT})$ imaging at the institute when recent outside scans were unavailable. If outside scans were available, these were reviewed by radiologists at the institute. Preoperative work-up also included all blood work-ups and relevant tumor markers. Patients selected for CRS + HIPEC by imaging routinely underwent laparoscopic staging prior to laparotomy. However, patients were selected for direct laparotomy if they had recent surgical evaluation prior to their referral to our program and the disease appeared amenable for CRS + HIPEC based on operative notes and cross sectional imaging. Patients were classified as extensive disease for exclusion from CRS + HIPEC for PCI scores higher than 11 for gastric cancer and 19 for colorectal and non-gastric primaries. In addition, patients with no suspicion for peritoneal carcinomatosis were also excluded from CRS + HIPEC.

\section{Technique for diagnostic laparoscopy}

The site of first port placement during diagnostic laparoscopy (DL) was decided at the surgeon's discretion based on imaging and clinical findings of the patient. The preferred technique for first creation of pneumoperitoneum was via the optical access technique in the left upper quadrant. In others, a Hasson's technique was used to establish pneumoperitoneum. After ruling out significant adhesions at the anterior abdominal wall, systematic visual examination of the abdomen was performed to generate the PCI score (complete laparoscopy) [7,22]. Systematic examination of the abdomen was then performed including special attention to peri-splenic, peri-hepatic, pelvic, omental bursa and bowel.

\section{Technique for hyperthermic intraperitoneal chemotherapy}

After cytoreduction, HIPEC was performed using the closed technique and dosing of therapy was based on standard published consensus guidelines [23].

\section{Subsequent management}

Patients excluded from CRS + HIPEC were either enrolled into a chemotherapy program with plan for future CRS + HIPEC or discharged to hospice or home care.

The first post-operative visit after CRS + HIPEC was planned at 6 weeks. Subsequent visits were planned to take place every 3 months for 2 years and subsequently, every 6 months for 3 years. The visits included physical examinations and lab chemistries including basic hematological parameters, blood chemistry, and tumor markers as appropriate. Imaging follow-up was performed using CT scans for the majority of the patients. Magnetic resonance imaging (MRI) and positron emission tomography (PET) scans were used in some patients, but were not routine. The most recent images were reviewed at every visit.

\section{Statistical analyses}

Statistical calculations were performed with STATA software Version 12.1 (StataCorp, Texas, USA). Continuous 
data are summarized as mean and standard deviation. Comparison was performed using Student's t-test for continuous data. Categorical variables were expressed as valid percentages and compared using the chi-squared test or Fisher's exact test as appropriate. The survival analysis was performed using Kaplan-Meier plots with log-rank analyses. Alpha was fixed at 0.05 for statistical significance.

\section{Results}

The baseline data and outcomes from CRS + HIPEC for the 101 patients studied are represented in Table 1 . The most prevalent primary was appendiceal (47.5\%), followed by colorectal (33.7\%) and gastric (6.9\%). The presentation of peritoneal carcinomatosis was synchronous in $22(21.8 \%)$ and metachronous in 79 (78.2\%) patients.

Laparoscopic evaluation (DL) was performed 73 times (12 as a separate procedure and 61 concurrent with planned CRS + HIPEC) in 70 patients, whereas 31 patients were selected for direct exploratory laparotomy (EL). Primary tumor resection prior to CRS was performed in 78 patients $(77.2 \%)$ overall: $56(80.0 \%)$ in the DL group versus $22(71.0 \%)$ receiving $\mathrm{EL}, P$ value $=$ 0.318 . The respective median intervals to second surgeries were 11.0 (interquartile range (IQR) 4.8 to 30.3 ) months for DL versus 19.8 (11.0 to 50.4) months for EL.

\section{Laparoscopy outcomes}

The technical details and outcomes of the DL, stratified by the timing of the procedure, are described in Table 2 and represented in Figure 1. The DL entry technique was optical trocar in $68.5 \%$ and Hasson trocar in $30.1 \%$ of the procedures. The left subcostal region was the favored site of entry in $60.3 \%$, while a midline periumbilical incision was used in $39.7 \%$ of the procedures. Complete laparoscopic evaluation was possible in 63 cases (of 73, 86.3\%) with 18 (of 73, 27.7\%) patients being excluded from laparotomy for CRS + HIPEC. The reason for exclusion was extensive disease (high PCI) in 11 cases and absence of carcinomatosis in 7. Laparoscopic CRS + HIPEC was performed in nine patients, with all patients achieving $\mathrm{CCO}$ cytoreduction and successful completion of HIPEC. Of the 46 patients who underwent laparotomy (4 with a separate laparoscopy and 42 with a concurrent laparoscopy), 38 were selected for CRS + HIPEC. HIPEC was not performed in six patients with extensive disease (who underwent resection of primary and cytoreduction without HIPEC) and two patients with no carcinomatosis (cases that were inadequately visualized in laparoscopy due to adhesions, these were converted to open). Therefore, CRS + HIPEC was successfully performed in $85.4 \%$ (DL, 47 of 55 not excluded by laparoscopy) compared to $74.2 \%$ (23 of 31 ,
Table 1 Baseline characteristics and outcomes of patients treated for peritoneal carcinomatosis $(\mathrm{N}=101)$

\begin{tabular}{lcc}
\hline Characteristic & Mean (SD) & $\begin{array}{c}\text { Frequency } \\
\mathbf{n}(\%)\end{array}$ \\
\hline Age of primary diagnosis in years & \\
Mean & $52.3(13.4)$ & \\
Sex & & \\
Male & $49(48.5)$ \\
Female & $52(51.5)$ \\
Primary Tumor Diagnosis & \\
Appendiceal & $48(47.5)$ \\
Colorectal & $34(33.7)$ \\
Gastric & $7(6.9)$ \\
Ovarian & $3(3.0)$ \\
Sarcoma & $3(3.0)$ \\
Others & $6(5.9)$
\end{tabular}

Grade of primary tumor $(n=85)$

Grade 1 (Well differentiated) $36(42.9)$

Grade 2 (Moderately differentiated) 23 (27.4)

Grade 3 (Poorly differentiated/signet ring) 25 (29.8)

Preoperative imaging

Computed tomography (CT) scan

Magnetic resonance imaging (MRI) scan 41 (40.6)

Positron emission tomography (PET) scan 62 (69.7)

Intraoperative PCI score $(n=74) \quad 15.5(11.7)$

HIPEC completed

$73(72.3)$

Completeness of cytoreduction $(n=65)$

CCO

$52(80.0)$

$\mathrm{CC} 1$

$\mathrm{CC} 2$

Length of stay

$11.3(9.1)$

Grade 3 to 5 complications 15 (20.5)

Chemotherapy received 46 (45.5)

Before CRS 16 (34.8)

After CRS 19 (41.3)

Both before and after CRS 11 (23.9)

Patients alive and under follow up 82 (81.2)

Status at last office visit $(n=82)$

No disease 31 (37.8)

Stable disease 24 (29.3)

Progressive disease

$27(32.9)$

CRS, cytoreductive surgery; HIPEC, hyperthermic intraperitoneal chemotherapy; $P C l$, peritoneal carcinomatosis index.

$P$ value $=0.20)$ in the EL group (Figure 2). The incidence of Clavien-Dindo grade 3 to 5 complications among those excluded from HIPEC were $0 \%$ with DL versus $10 \%$ with EL $(P$ value $=0.2)$. 
Table 2 Technical details and outcomes of diagnostic laparoscopy (DL) stratified by the timing of the procedure with respect to planned laparotomy for cytoreductive surgery and hyperthermic intraperitoneal chemotherapy $\left(\right.$ CRS + HIPEC) ${ }^{a}$

\begin{tabular}{|c|c|c|}
\hline Characteristic & $\begin{array}{l}\text { Procedure done concurrently with } \\
\text { planned laparotomy for CRS + HIPEC } \\
\qquad(n=61)\end{array}$ & $\begin{array}{l}\text { Procedure done separately } \\
\text { prior to laparotomy for CRS + } \\
\text { HIPEC }(\mathrm{N}=12)\end{array}$ \\
\hline \multicolumn{3}{|l|}{ Entrance technique } \\
\hline Visual entry system & $43(70.5)$ & 8 \\
\hline Hasson trocar & $18(29.5)$ & 4 \\
\hline \multicolumn{3}{|l|}{ Site entry } \\
\hline Left upper quadrant & $38(62.3)$ & 6 \\
\hline Midline periumbilical & $23(37.7)$ & 6 \\
\hline $\begin{array}{l}\text { Laparoscopy converted to open (inadequate visualization due } \\
\text { to adhesions) }\end{array}$ & $10(16.4)$ & 0 \\
\hline Excluded from laparotomy for CRS + HIPEC after adequate evaluation & $10(19.6)$ & 8 \\
\hline \multicolumn{3}{|l|}{ Reason for exclusion } \\
\hline Extensive disease & 5 & 6 \\
\hline No carcinomatosis & 5 & 2 \\
\hline Completed HIPEC & $44(86.3)$ & $\begin{array}{l}3 \text { (1 Patient underwent CRS only } \\
\text { due to poor functional status) }\end{array}$ \\
\hline Open HIPEC & 35 & 3 \\
\hline Laparoscopic HIPEC & 9 & 0 \\
\hline $\begin{array}{l}\text { Excluded from HIPEC at laparotomy ( } n=42: 32 \text { cases selected after } \\
\text { complete laparoscopic evaluation and } 10 \text { cases of laparoscopic } \\
\text { conversion to open) }\end{array}$ & 7 & 0 \\
\hline \multicolumn{3}{|l|}{ Reason for exclusion } \\
\hline Extensive disease & 5 & \\
\hline $\begin{array}{l}\text { No carcinomatosis (Cases converted from laparoscopy due to } \\
\text { inadequate evaluation) }\end{array}$ & 2 & \\
\hline Delayed HIPEC for those excluded at laparoscopy & 2 & 3 \\
\hline
\end{tabular}

${ }^{a}$ Two patients underwent both concurrent and separate DL.

Of the 11 cases with extensive disease that were excluded from CRS + HIPEC by laparoscopy, 5 received delayed CRS + HIPEC following systemic chemotherapy and down-staging of the disease. At a mean follow-up of $9.1 \pm 8$ months, 82 patients were alive. At the latest office visit, $31(37.8 \%)$ had no disease while 24(29.3\%) had stable disease. Progressive disease was detected in $27(32.9 \%)$ patients. There was no port-site recurrences at current follow-up.

\section{Discussion}

CRS + HIPEC is an evolving treatment option for treatment of patients with peritoneal carcinomatosis and has been shown to significantly improve survival in multiple studies [11,24-26]. The present study demonstrates the feasibility and safety of laparoscopy in the treatment paradigm of patients with suspected peritoneal disease.

The maximum impact of the laparoscopy is in reducing non-therapeutic laparotomies for patients with no disease (38.9\%) and in assessing patients with high burden of disease with high grade histology (61.1\%), allowing the disease to be down-staged with systemic chemotherapy. An important finding was that many patients with extensive disease who were excluded by DL were able to subsequently undergo CRS + HIPEC after the downgrading of their disease by chemotherapy (5 out 11 patients with extensive disease excluded by DL) (Figure 1).

Based on our institutional practice, a diagnostic laparoscopy was not routinely utilized in patients with low-grade disease such as DPAM unless concerns for completion of cytoreduction were present, or in patients with bowel obstruction and in patients with a hostile abdomen, which does constitute a significant proportion of patients in a practice at a peritoneal surface malignancy center.

Oncological concerns of a laparoscopy include incomplete assessment of the peritoneal cavity and port site recurrences. In our study, we found that we were able to assess the PCI score more as a threshold rather than for accuracy. We recognize that open assessment of the 


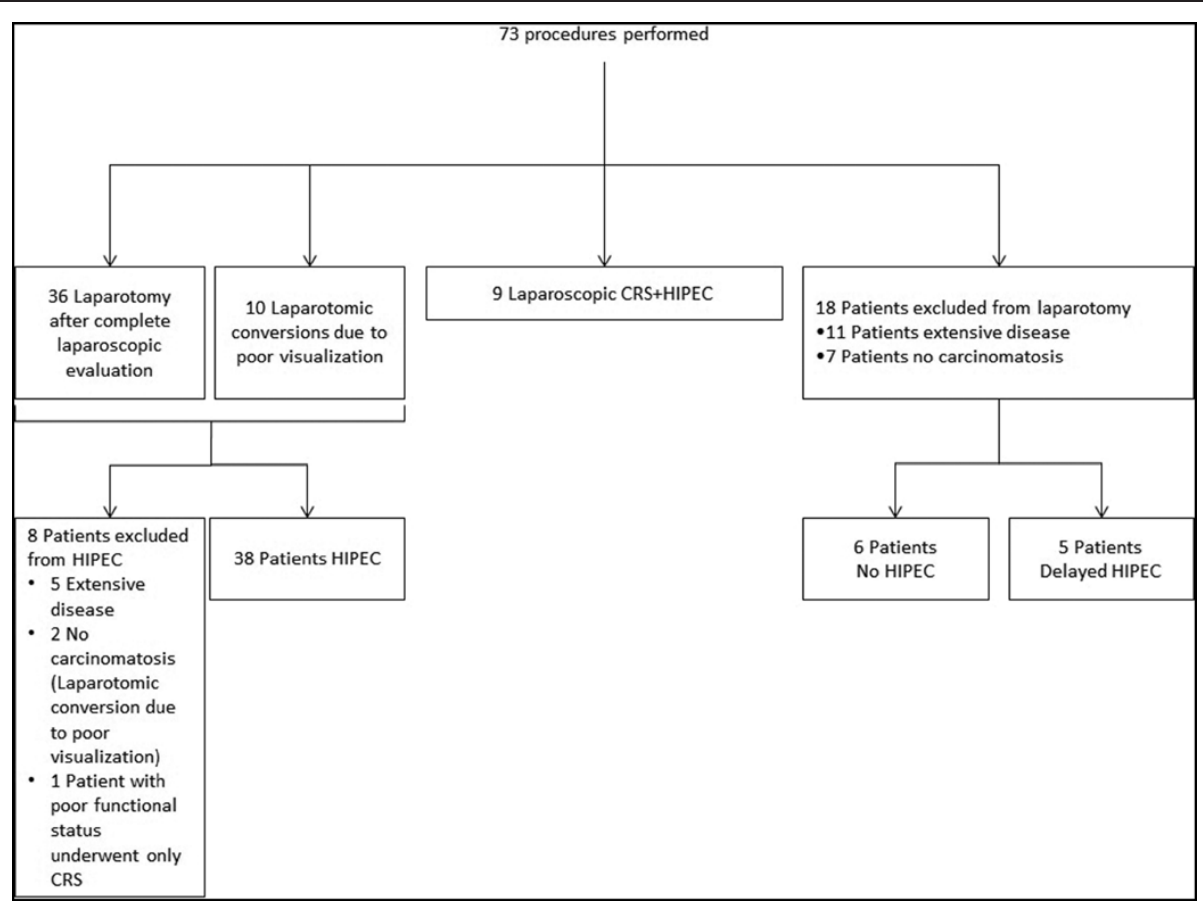

Figure 1 Flowchart depicting the outcomes of diagnostic laparoscopy (DL). CRS, cytoreductive surgery; HIPEC, hyperthermic intraperitoneal chemotherapy.

peritoneum may be superior to laparoscopy especially for the right hemidiaphragm, omental bursa and the pelvis, yet believe that an assessment of high burden of disease is easily feasible with a laparoscopy.

Patients selected for HIPEC often belong to advanced age groups associated with poor performance status resulting in increased morbidity rates $[27,28]$. The peritoneal carcinomatosis index is a well-validated tool of prognostic significance for assessing the burden of peritoneal disease and is used for selecting patients for CRS + HIPEC [7]. The sensitivity of cross-sectional imaging to accurately describe peritoneal carcinomatosis remains low despite the recent advancements [16,20,29,30]. As many as 20 to $40 \%$ of the patients considered eligible for HIPEC based on imaging are excluded from HIPEC at laparotomy $[17,18]$. Such non-therapeutic laparotomies may adversely affect the outcome of this vulnerable population with short life period, as well as affecting the physician patient relationship, thereby supporting the use of a diagnostic laparoscopy.

The use of laparoscopy in PC has hitherto remained limited due to technical concerns that the adhesions from cancer and past surgeries may hinder adequate assessment and possibly result in an increased rate of

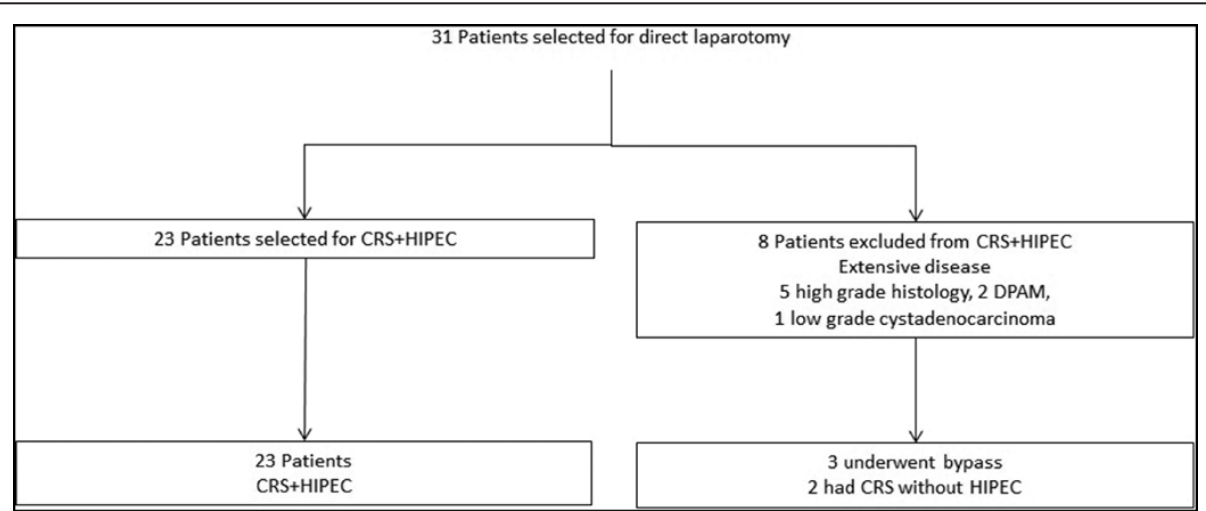

Figure 2 Flowchart depicting the outcomes of patients who did not undergo diagnostic laparoscopy. CRS, Cytoreductive surgery; HIPEC, hyperthermic intraperitoneal chemotherapy; DPAM, disseminated peritoneal adenomucinosis. 
complications $[21,31]$. In the present study, DL was successful in a majority (89.2\%) of the patients and was not associated with any complications.

All patients underwent advanced imaging (triple phase helical CT scans/MRI) routinely before the surgical evaluation for HIPEC. All outside images were also reviewed at the institute. Therefore the high rate of exclusion from CRS + HIPEC (28.4\%) reflects the low fidelity of imaging for classifying patients for CRS + HIPEC. This is lower than the exclusion rates reported for gallbladder cancer and also for pancreatic cancer in the preneoadjuvant paradigm and could be attributed to the good quality cross-sectional imaging $[16,19,20,30,32,33]$. The fact that many of these patients were referred from outside following incidental diagnosis for peritoneal carcinomatosis during surgery for primary tumor and had adequate EL evaluation may also have contributed to the lower rate of exclusion from curative surgery.

Use of laparoscopy for staging may permit its extension to performing HIPEC when feasible. In the group of patients evaluated for this study, CC0 cytoreduction followed by successful HIPEC was achieved laparoscopically in nine patients. Other groups have also reported the feasibility of laparoscopic HIPEC [21]. Since most of the patients would need to continue chemotherapy after HIPEC, a laparoscopic procedure may permit lowering of this convalescent period to chemotherapy with potential impact on the outcome.

The routine use of laparoscopy has significant financial implications as well since the laparoscopy adds to the overall cost of therapy. In a previous paper, we studied the cost effectiveness of laparoscopy in pancreatic cancer and demonstrated that laparoscopy was cost-effective at high probability for exclusion from resection [33]. This is directly related to pretest probability for exclusion as well as the sensitivity of laparoscopy. which may be subjective to the surgeon and influenced by the experience. The use of imaging modalities like diffusion-weighted MRI and PET scanning for the identification of peritoneal disease is evolving but may increase the cost differential dramatically. These modalities were used during diagnosis and follow-up of the patients, but the sensitivity of these modalities is yet to be validated. The radiological evaluation permits only anatomic characterization of the extent of the disease whereas laparoscopy permits pathological diagnosis as well with potential prognostic value.

A potential limitation of the study is that the sample was small in size and taken from a single institution. The study may suffer from confounding biases inherent to the observational study design. In addition, we did not formally correlate the laparoscopic PCI with an open PCI, although we believe that a laparoscopic PCI would under-stage rather than over-stage someone with disease and thus not alter the algorithms proposed.

\section{Conclusions}

Laparoscopy is feasible in patients with peritoneal carcinomatosis who are expected to undergo cytoreductive surgery. It can filter out ineligible patients with either no carcinomatosis or extensive disease precluding complete cytoreduction during CRS + HIPEC. Technical and oncological concerns of performing a laparoscopy were underwhelming based on our study.

\section{Abbreviations}

CC $(0,1,2)$ : completeness of cytoreduction; CRS + HIPEC: cytoreductive surgery and hyperthermic intraperitoneal chemotherapy; CT: computerized tomography; DL: diagnostic laparoscopy; DPAM: disseminated peritoneal adenomucinosis; EL: exploratory laparotomy; IQR: interquartile range; MRI: magnetic resonance imaging; PC: peritoneal carcinomatosis; PCl: peritoneal carcinomatosis index; PET: positron emission tomography.

\section{Competing interests}

This study was supported by the Sebastian Raclaw Abdominal Cancer Fund. The study sponsor had no involvement in the study. The other authors declare that they have no relevant disclosures to make.

\section{Authors' contribution}

$T T J, A Z, K K T$ : Responsible for design of study question, hypothesis and study design. TTJ, AZ, AS: Collection of data. TTJ, AZ, KKT: Analysis of data. SP, KKT, TCG: Interpretation of data. TJ, KKT: Drafting of manuscript. SP, TCG, KKT: Revision of manuscript. All authors read and approved the final manuscript.

\section{Acknowledgements}

This study was presented at the annual conference of the Wisconsin Chapter of the American College of Surgeons at Kohler, WI (8 to 9 November 2013) and 9th International Symposium on Regional Cancer Therapies at Steamboat Springs, Colorado (15 to 17 February 2014).

\section{Author details}

${ }^{1}$ Division of Surgical Oncology, Department of Surgery, Medical College of Wisconsin, 9200 W Wisconsin Ave, Milwaukee, WI 53226, USA. ²Division of Surgical Oncology, Department of Surgery, Loyola University Medical Center, 2160 South 1st Avenue, Maywood, IL, USA.

Received: 13 May 2014 Accepted: 20 July 2014

Published: 21 August 2014

\section{References}

1. Jayne DG, Fook S, Loi C, Seow-Choen F: Peritoneal carcinomatosis from colorectal cancer. Br J Surg 2002, 89:1545-1550.

2. Segelman J, Granath F, Holm T, Machado M, Mahteme H, Martling A: Incidence, prevalence and risk factors for peritoneal carcinomatosis from colorectal cancer. Br J Surg 2012, 99:699-705.

3. Chu DZ, Lang NP, Thompson C, Osteen PK, Westbrook KC: Peritoneal carcinomatosis in nongynecologic malignancy. A prospective study of prognostic factors. Cancer 1989, 63:364-367.

4. Sadeghi B, Arvieux C, Glehen O, Beaujard AC, Rivoire M, Baulieux J, Fontaumard E, Brachet A, Caillot JL, Faure JL, Porcheron J, Peix JL, Francois $Y$, Vignal J, Gilly FN: Peritoneal carcinomatosis from non-gynecologic malignancies: results of the EVOCAPE 1 multicentric prospective study. Cancer 2000, 88:358-363.

5. Elias D, Lefevre JH, Chevalier J, Brouquet A, Marchal F, Classe JM, Ferron G, Guilloit JM, Meeus P, Goere D, Bonastre J: Complete cytoreductive surgery plus intraperitoneal chemohyperthermia with oxaliplatin for peritoneal carcinomatosis of colorectal origin. J Clin Oncol 2009, 27:681-685.

6. Sugarbaker PH: Peritoneal surface oncology: review of a personal experience with colorectal and appendiceal malignancy. Tech Coloproctol 2005, 9:95-103.

7. Harmon RL, Sugarbaker PH: Prognostic indicators in peritoneal carcinomatosis from gastrointestinal cancer. Int Semin Surg Oncol 2005, $2: 3$ 
8. Yonemura $\mathrm{Y}$, Canbay $\mathrm{E}$, Ishibashi $\mathrm{H}$ : Prognostic factors of peritoneal metastases from colorectal cancer following cytoreductive surgery and perioperative chemotherapy. Scientific World J 2013, 2013:978394.

9. Van Sweringen HL, Hanseman DJ, Ahmad SA, Edwards MJ, Sussman JJ: Predictors of survival in patients with high-grade peritoneal metastases undergoing cytoreductive surgery and hyperthermic intraperitoneal chemotherapy. Surgery 2012, 152:617-624. discussion 624-615.

10. Tentes AA, Tripsiannis G, Markakidis SK, Karanikiotis CN, Tzegas G, Georgiadis G, Avgidou K: Peritoneal cancer index: a prognostic indicator of survival in advanced ovarian cancer. Eur J Surg Oncol 2003, 29:69-73.

11. Yang XJ, Huang CQ, Suo T, Mei L, Yang GL, Cheng FL, Zhou YF, Xiong B, Yonemura Y, Li Y: Cytoreductive surgery and hyperthermic intraperitoneal chemotherapy improves survival of patients with peritoneal carcinomatosis from gastric cancer: final results of a phase III randomized clinical trial. Ann Surg Oncol 2011, 18:1575-1581

12. Bakrin N, Gilly FN, Baratti D, Bereder JM, Quenet F, Lorimier G, Mohamed F, Elias D, Glehen O: Primary peritoneal serous carcinoma treated by cytoreductive surgery combined with hyperthermic intraperitoneal chemotherapy. A multi-institutional study of 36 patients. Eur J Surg Oncol 2013, 39:742-747.

13. Quenet F, Goere D, Mehta SS, Roca L, Dumont F, Hessissen M, Saint-Aubert B, Elias D: Results of two bi-institutional prospective studies using intraperitoneal oxaliplatin with or without irinotecan during HIPEC after cytoreductive surgery for colorectal carcinomatosis. Ann Surg 2011, 254:294-301.

14. Wu XJ, Yuan P, Li ZY, Bu ZD, Zhang LH, Wu AW, Zong XL, Li SX, Shan F, Ji X,

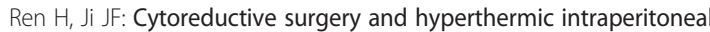
chemotherapy improves the survival of gastric cancer patients with ovarian metastasis and peritoneal dissemination. Tumour Biol 2013, 34:463-469

15. Yonemura $Y$, Elnemr A, Endou Y, Ishibashi H, Mizumoto A, Miura M, Li Y: Effects of neoadjuvant intraperitoneal/systemic chemotherapy (bidirectional chemotherapy) for the treatment of patients with peritoneal metastasis from gastric cancer. Int J Surg Oncol 2012, 2012:148420.

16. Society of American Gastrointestinal and Endoscopic Surgeons: Guidelines for Diagnostic Laparoscopy. Updated April 2010. [http://www. sages.org/publications/guidelines/guidelines-for-diagnostic-laparoscopy/]

17. Iversen LH, Rasmussen PC, Laurberg S: Value of laparoscopy before cytoreductive surgery and hyperthermic intraperitoneal chemotherapy for peritoneal carcinomatosis. Br J Surg 2013, 100:285-292.

18. Pomel C, Appleyard TL, Gouy S, Rouzier R, Elias D: The role of laparoscopy to evaluate candidates for complete cytoreduction of peritoneal carcinomatosis and hyperthermic intraperitoneal chemotherapy. Eur J Surg Oncol 2005, 31:540-543.

19. D'Angelica M, Fong Y, Weber S, Gonen M, DeMatteo RP, Conlon K, Blumgart $\mathrm{LH}$, Jarnagin WR: The role of staging laparoscopy in hepatobiliary malignancy: prospective analysis of 401 cases. Ann Surg Oncol 2003, 10:183-189.

20. Chang L, Stefanidis D, Richardson WS, Earle DB, Fanelli RD: The role of staging laparoscopy for intraabdominal cancers: an evidence-based review. Surg Endosc 2009, 23:231-241.

21. Sommariva A, Zagonel V, Rossi CR: The role of laparoscopy in peritoneal surface malignancies selected for hyperthermic intraperitoneal chemotherapy (HIPEC). Ann Surg Oncol 2012, 19:3737-3744.

22. Jacquet $\mathrm{P}$, Sugarbaker $\mathrm{PH}$ : Clinical research methodologies in diagnosis and staging of patients with peritoneal carcinomatosis. Cancer Treat Res 1996, 82:359-374.

23. Turaga K, Levine E, Barone R, Sticca R, Petrelli N, Lambert L, Nash G, Morse M, Adbel-Misih R, Alexander HR, Attiyeh F, Bartlett D, Bastidas A, Blazer T, Chu Q, Chung K, Dominguez-Parra L, Espat NJ, Foster J, Fournier K, Garcia R, Goodman M, Hanna N, Harrison L, Hoefer R, Holtzman M, Kane J, Labow D, Li B, Lowy A, et al: Consensus Guidelines from the American Society of Peritoneal Surface Malignancies on standardizing the delivery of Hyperthermic Intraperitoneal Chemotherapy (HIPEC) in Colorectal Cancer Patients in the United States. Ann Surg Oncol 2013.

24. Verwaal VJ, van Ruth S, Witkamp A, Boot H, van Slooten G, Zoetmulder FA Long-term survival of peritoneal carcinomatosis of colorectal origin. Ann Surg Oncol 2005, 12:65-71.

25. Verwaal VJ, Bruin S, Boot H, van Slooten G, van Tinteren H: 8-year follow-up of randomized trial: cytoreduction and hyperthermic intraperitoneal chemotherapy versus systemic chemotherapy in patients with peritoneal carcinomatosis of colorectal cancer. Ann Surg Oncol 2008, 15:2426-2432.

26. Glehen O, Kwiatkowski F, Sugarbaker PH, Elias D, Levine EA, De Simone M, Barone R, Yonemura Y, Cavaliere F, Quenet F, Gutman M, Tentes AA Lorimier G, Bernard JL, Bereder JM, Porcheron J, Gomez-Portilla A, Shen P, Deraco M, Rat P: Cytoreductive surgery combined with perioperative intraperitoneal chemotherapy for the management of peritoneal carcinomatosis from colorectal cancer: a multi-institutional study. J Clin Oncol 2004, 22:3284-3292.

27. Votanopoulos Kl, Newman NA, Russell G, Ihemelandu C, Shen P, Stewart JH, Levine EA: Outcomes of Cytoreductive Surgery (CRS) with Hyperthermic Intraperitoneal Chemotherapy (HIPEC) in patients older than 70 Years; survival benefit at considerable morbidity and mortality. Ann Surg Oncol 2013, 20(11):3497-3503.

28. Tabrizian P, Jibara G, Shrager B, Franssen B, Yang MJ, Sarpel U, Hiotis S, Labow D: Outcomes for cytoreductive surgery and hyperthermic intraperitoneal chemotherapy in the elderly. Surg Oncol 2013, 22(3):184-189.

29. Callery MP, Chang KJ, Fishman EK, Talamonti MS, William Traverso L, Linehan DC: Pretreatment assessment of resectable and borderline resectable pancreatic cancer: expert consensus statement. Ann Surg Oncol 2009, 16:1727-1733.

30. Catheline JM, Turner R, Rizk N, Barrat C, Champault G: The use of diagnostic laparoscopy supported by laparoscopic ultrasonography in the assessment of pancreatic cancer. Surg Endosc 1999, 13:239-245.

31. Yan TD, Morris DL, Shigeki K, Dario B, Marcello D: Preoperative investigations in the management of peritoneal surface malignancy with cytoreductive surgery and perioperative intraperitoneal chemotherapy: Expert consensus statement. J Surg Oncol 2008, 98:224-227.

32. Jayakrishnan TT, Nadeem H, Groeschl RT, George B, Thomas JP, Ritch PS, Christians KK, Tsai S, Evans DB, Pappas SG, Gamblin TC, Turaga KK: Diagnostic laparoscopy should be performed before definitive resection for pancreatic cancer: a financial argument. In Annual Meeting of the Americas Hepato-Pancreato-Biliary Association; Miami, FL. 2014

33. Nadeem H, Jayakrishnan $\Pi$, Groeschl RT, Zacharias A, Clark Gamblin T, Turaga KK: Cost effectiveness of routine Laparoscopic ultrasound for assessment of resectability of gallbladder cancer. Ann Surg Oncol 2014, 21(7):2413-2419.

\section{doi:10.1186/1477-7819-12-270}

Cite this article as: Jayakrishnan et al:: Role of laparoscopy in patients with peritoneal metastases considered for cytoreductive surgery and hyperthermic intraperitoneal chemotherapy (HIPEC). World Journal of Surgical Oncology 2014 12:270

\section{Submit your next manuscript to BioMed Central and take full advantage of:}

- Convenient online submission

- Thorough peer review

- No space constraints or color figure charges

- Immediate publication on acceptance

- Inclusion in PubMed, CAS, Scopus and Google Scholar

- Research which is freely available for redistribution 